\title{
Fixed point indices of homeomorphisms defined on the torus
}

\author{
Hisao SHIRAKI \\ (Received May 14, 2001; Revised January 25, 2002)
}

\begin{abstract}
Let $h: T^{2} \rightarrow T^{2}$ be a homeomorphism on the 2-dimensional torus $T^{2}$ isotopic to the identity map. We assume that two fixed points of $h$ have been found. Then, we classify all the other fixed points into Nielsen classes and find some relations among fixed point indices of $h$.
\end{abstract}

Key words: fixed point index, Nielsen class, generalized Lefschetz number, braid.

\section{Introduction}

Let $X$ be a compact connected polyhedron and $f: X \rightarrow X$ a continuous map. Denote by $\operatorname{Fix}(f)$ the set of fixed points of $f$. The generalized Lefschetz number $L(f)$ is a topological invariant which is useful to study fixed points.

Let $h: T^{2} \rightarrow T^{2}$ be a homeomorphism on the 2-dimensional torus $T^{2}$ isotopic to the identity map $i d$. In this paper we consider its fixed point set. In this case, the generalized Lefschetz number $L(h)$ vanishes and provides no information on fixed points. One of the methods extracting some information on fixed points is to consider the restriction of $h$ to some complement $T^{2}-C$, where $C$ is a finite set of fixed points. However the set $T^{2}-C$ is not compact, and the theory of the generalized Lefschetz number cannot be applied. So we need to compactify the map $h: T^{2} \rightarrow$ $T^{2}$ to a map $f: X \rightarrow X$, which is called the blow-up of $h[3$, p. 24]. By this compactification, there may arise some fixed points of $f$ which are not fixed points of the original map $h$. However, these extra fixed points are determined entirely by the derivatives of $h$ on $C$ if $h$ is differentiable on $C$, and we can obtain some information on fixed points of the original map $h$ by investigating fixed points of $f$.

In [9], the author considered the case of $\sharp C=2$ and showed that a reduced form $\widehat{L}(f)$ of $L(f)$, which is a polynomial with one variable, is a symmetric polynomial under a certain condition. This tells us that the fixed 
point indices obey a restriction which is unexpected from the definition of $\widehat{L}(f)$ itself. It is known that the homomorphism on the fundamental group of the punctured torus induced by $h$ can be identified with a braid on two strings. Therefore the induced homomorphism is written as a product of certain braids $\rho$ and $\tau$. The paper [9] treated only the special case where it is expressed as the commutator $\tau^{m} \rho^{n} \tau^{-m} \rho^{-n}(m, n \in \mathbf{Z})$.

The purpose of the present paper is to show that the same result as in [9] holds also in the general case. As in [9], our result is obtained not by a geometric consideration but by the algebraic calculation using the result of Huang and Jiang on the abelianized generalized Lefschetz number $L(f)^{\mathrm{Ab}}$ [6]. Huang and Jiang derived a method of calculating $L(f)^{\mathrm{Ab}}$ in the case of a compact surface $X$ from the result of Fadell and Husseini [5], which gives a method of calculating $L(f)$. The proof of our result uses their method of the computation of $L(f)^{\mathrm{Ab}}$.

\section{Definition of generalized Lefschetz number}

Let $X$ be a compact connected polyhedron, and $f: X \rightarrow X$ a continuous map.

Definition 1 We shall classify $\operatorname{Fix}(f)$ by the following equivalence relation: $x, y \in \operatorname{Fix}(f)$ are said to be Nielsen equivalent if there exists a path $q$ from $x$ to $y$ such that $q$ and $f \circ q$ are homotopic relative to the end points $\{x, y\}$.

Choose a base point $x_{0} \in X$ and a path $w$ from $x_{0}$ to $f\left(x_{0}\right)$. Let $\pi_{1}\left(X, x_{0}\right)$ be the fundamental group of $X$ relative to the base point $x_{0}$, and let $f_{\pi}: \pi_{1}\left(X, x_{0}\right) \rightarrow \pi_{1}\left(X, x_{0}\right)$ be the composition:

$$
\pi_{1}\left(X, x_{0}\right) \stackrel{f_{*}}{\rightarrow} \pi_{1}\left(X, f\left(x_{0}\right)\right) \stackrel{w_{*}}{\rightarrow} \pi_{1}\left(X, x_{0}\right) .
$$

Definition 2 Two elements $\alpha, \beta \in \pi_{1}\left(X, x_{0}\right)$ are said to be Reidemeister equivalent if there is a $\gamma \in \pi_{1}\left(X, x_{0}\right)$ such that $\beta=f_{\pi}(\gamma) \alpha \gamma^{-1}$.

Thus $\pi_{1}\left(X, x_{0}\right)$ is devided into Reidemeister equivalence classes. Let $R(f)$ denote the set of Reidemeister equivalence classes, and $\mathbf{Z} R(f)$ the free abelian group generated by the set $R(f)$.

Definition 3 For $x \in \operatorname{Fix}(f)$, take a path $\ell$ from $x_{0}$ to $x$. The Reidemeister equivalence class represented by $\left[w(f \circ \ell) \ell^{-1}\right] \in \pi_{1}\left(X, x_{0}\right)$ is called the 
coordinate of $x$, and is denoted by $R(x)$.

Note that $R(x)$ is evidently independent of the choice of $\ell$. It is easy to see that two fixed points are in the same Nielsen class if and only if they have the same coordinate. Thus for a Nielsen class, its coordinate can be defined.

Definition 4 For $\alpha \in \pi_{1}\left(X, x_{0}\right)$, let

$$
\operatorname{Fix}_{[\alpha]}(f)=\{x \in \operatorname{Fix}(f) \mid R(x)=[\alpha]\} .
$$

The generalized Lefschetz number $L(f)$ is defined as

$$
L(f)=\sum_{[\alpha] \in R(f)} \operatorname{ind}\left(\operatorname{Fix}_{[\alpha]}(f)\right)[\alpha] \in \mathbf{Z} R(f),
$$

where ind $\left(\operatorname{Fix}_{[\alpha]}(f)\right)$ is the fixed point index of $\operatorname{Fix}_{[\alpha]}(f)$. For the definition of the fixed point index, see [4], [7].

From this definition, it is clear that the number of non-zero terms in $L(f)$ is a lower bound for the number of fixed points of $f$.

The notations above have a homological version obtained by abelianizing $\pi_{1}\left(X, x_{0}\right)$ into the 1-dimensional homlogy group $H_{1}(X)$.

Definition 5 We shall classify $\operatorname{Fix}(f)$ by the following equivalence relation: $x, y \in \operatorname{Fix}(f)$ are said to be abelianized Nielsen equivalent if there exists a path $q$ from $x$ to $y$ such that $\left[(f \circ q) q^{-1}\right]$ is the zero element of $H_{1}(X)$.

Let $x \in \operatorname{Fix}(f)$. We choose a path $\ell$ from $x_{0}$ to $x$. Then we can identify the abelianized Nielsen class $[x]$ with an element $\left[w(f \circ \ell) \ell^{-1}\right]$ of $\operatorname{Coker}\left(f_{*}-\right.$ $i d)$ naturally, where $f_{*}$ is the homomorphism on $H_{1}(X)$ induced by $f$ and Coker $\left(f_{*}-i d\right)=H_{1}(X) / \operatorname{Im}\left(f_{*}-i d\right)$. This correspondence is evidently independent of the choice of $\ell$.

Definition 6 For $x \in \operatorname{Fix}(f)$, define $R(x)^{\mathrm{Ab}}=\left[w(f \circ \ell) \ell^{-1}\right] \in \operatorname{Coker}\left(f_{*}\right.$ $i d)$. We call $R(x)^{\mathrm{Ab}}$ the abelianized coordinate of $x$.

Definition 7 For $\gamma \in \operatorname{Coker}\left(f_{*}-i d\right)$, let

$$
\operatorname{Fix}_{\gamma}(f)=\left\{x \in \operatorname{Fix}(f) \mid R(x)^{\mathrm{Ab}}=\gamma\right\} \text {. }
$$


Define the abelianization $L(f)^{\mathrm{Ab}}$ of $L(f)$ as

$$
L(f)^{\mathrm{Ab}}=\sum_{\gamma \in \operatorname{Coker}\left(f_{*}-i d\right)} \operatorname{ind}\left(\operatorname{Fix}_{\gamma}(f)\right) \gamma \in \mathbf{Z} \operatorname{Coker}\left(f_{*}-i d\right),
$$

where $\mathbf{Z} \operatorname{Coker}\left(f_{*}-i d\right)$ is the integral group ring of $\operatorname{Coker}\left(f_{*}-i d\right)$. From this definition, it is clear that $L(f)^{\mathrm{Ab}}$ is a Laurant polynomial.

\section{Statement of result}

Let $h: T^{2} \rightarrow T^{2}$ be a homeomorphism isotopic to $i d$, and let $x_{1}, x_{2}$ be distinct fixed points of $h$. Suppose that $h$ is differentiable at $x_{i}$, and the derivatives $\mathrm{D} h\left(x_{i}\right)$ are non-singular $(i=1,2)$. Set $C=\left\{x_{1}, x_{2}\right\}$ and $M=$ $T^{2}-C$. Then we can consider $h: M \rightarrow M$. Let $X$ be the compactification of $M$ obtained from $T^{2}$ by blowing up each $x_{i}$ to a circle $S_{i}(i=1,2)$, and $f: X \rightarrow X$ the extention of $h[3$, p. 24].

Now, pick a base point $x_{0}$ for $M$, and let $a_{1}, a_{2}, b, c$ be the elements of $\pi_{1}\left(M, x_{0}\right)$ indicated in Figure 1.

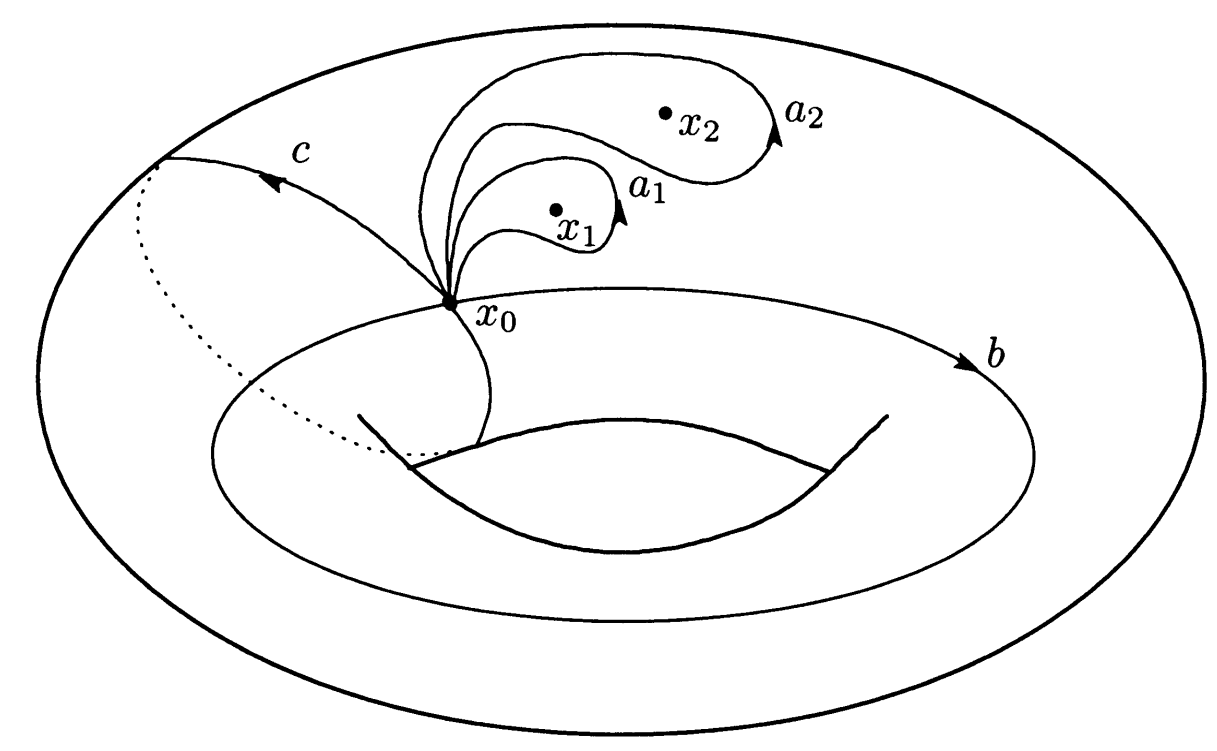

Fig. 1.

We use the commutator notation $[\alpha, \beta]=\alpha \beta \alpha^{-1} \beta^{-1}$ in groups. We have that $a_{1}=[b, c] a_{2}^{-1}$ and that $\pi_{1}\left(M, x_{0}\right)$ is a free group of rank 3 generated by $a_{2}, b, c$. Therefore the 1-dimensional homology group $H_{1}(M)$ is an abelian group generated by $a_{2}, b, c$, and we have a relation $a_{1}+a_{2}=0$. Let $\Lambda$ denote the group ring $\mathbf{Z} H_{1}(M)$. 
We use the same notation $h_{*}$ for the extention of $h_{*}: H_{1}(M) \rightarrow H_{1}(M)$ to $\Lambda$. Since $X$ is the compactification of $M$, we can identify $\pi_{1}\left(X, x_{0}\right)$ with $\pi_{1}\left(M, x_{0}\right)$ naturally. Then the homomorphism $f_{*}: H_{1}(X) \rightarrow H_{1}(X)$ is identified with the homomorphism $h_{*}: H_{1}(M) \rightarrow H_{1}(M)$. Then $H_{1}(X)$ is generated by $a_{2}, b, c$, and we have

$$
\operatorname{Coker}\left(f_{*}-i d\right)=\mathbf{Z} a_{2} \oplus \mathbf{Z} b \oplus \mathbf{Z} c / \operatorname{Im}\left(f_{*}-i d\right) .
$$

Now let us recall some facts about braids on the torus. The braids $\rho_{i}, \tau_{i}$ $(i=1,2)$ used below are indicated in Figure 2.

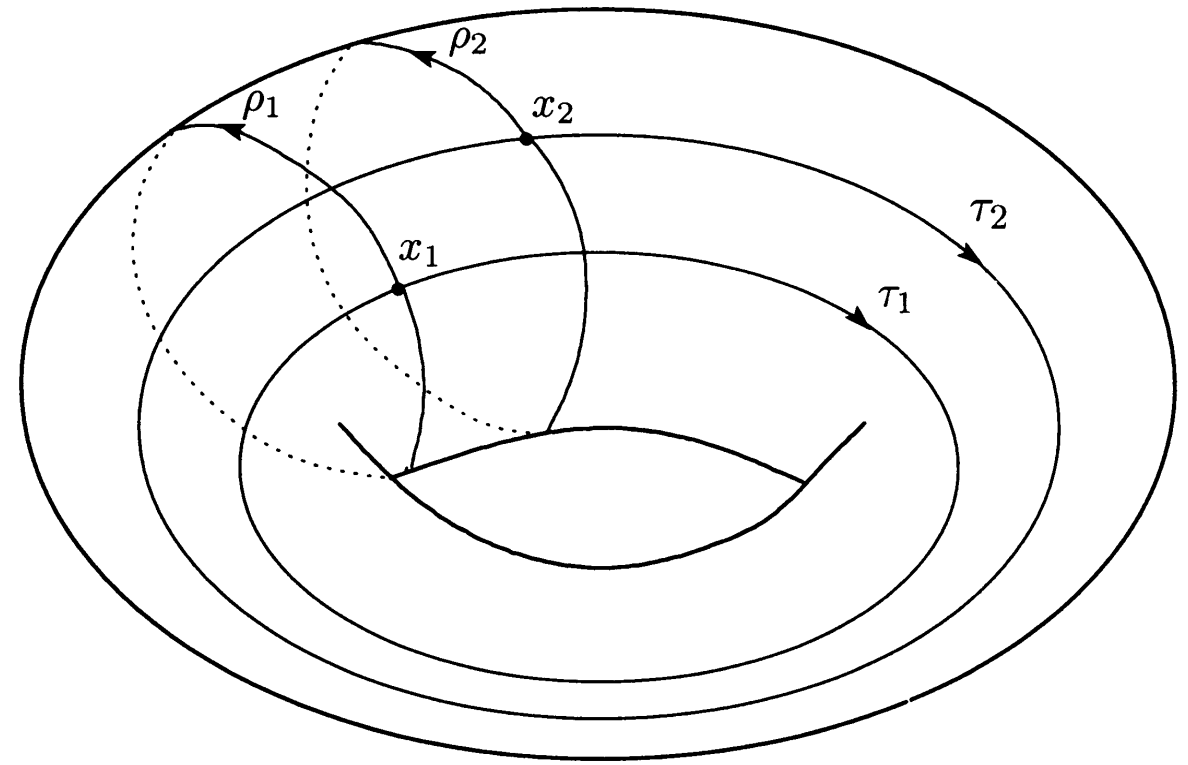

Fig. 2 .

Proposition 1 (Birman [1]) The pure 2-braid group on $T^{2}$ admits the following presentation:

Generators: $\rho_{1}, \rho_{2}, \tau_{1}, \tau_{2}$.

Relations: $\left[\rho_{1}, \rho_{2}\right]=\left[\tau_{1}, \tau_{2}\right]=1, A_{12}=\left[\tau_{2}^{-1}, \rho_{1}\right], A_{12}^{-1}=\left[\rho_{2}^{-1}, \tau_{1}\right]$,

$$
\begin{aligned}
& A_{12}^{-1}=\left(\tau_{1} \tau_{2}\right) A_{12}^{-1}\left(\tau_{2}^{-1} \tau_{1}^{-1}\right), A_{12}=\left(\rho_{1} \rho_{2}\right) A_{12}\left(\rho_{2}^{-1} \rho_{1}^{-1}\right), \\
& \text { where } A_{12}=\left[\tau_{1}, \rho_{1}\right] .
\end{aligned}
$$

Now we choose an isotopy $\left\{h_{t}\right\}: T^{2} \rightarrow T^{2}$, where $h_{0}=i d, h_{1}=h$. Then $\left\{h_{t}\right\}$ determines a subset $h_{t}(C)=\left\{h_{t}\left(x_{1}\right), h_{t}\left(x_{2}\right)\right\}$ of $T^{2}$ with 2 points for each $t$. The subset $h_{t}(C)$ determines a braid [2], [8]. The braid represented by $h_{t}(C)$ depends on the choice of an isotopy $\left\{h_{t}\right\}$. We can choose the 
isotopy $\left\{h_{t}\right\}$ so as to satisfy $h_{t}\left(x_{2}\right)=x_{2}$ for any $t(0 \leq t \leq 1)$. Let $\sigma_{C}$ denote the braid represented by $h_{t}(C)=\left\{h_{t}\left(x_{1}\right), x_{2}\right\}$. It is easy to see that the braid $\sigma_{C}$ is uniquely determined. Moreover, it is written as a product of $\rho_{1}^{ \pm 1}$ or $\tau_{1}^{ \pm 1}$ uniquely.

For brevity, we shall write $a=a_{2}, \rho=\rho_{1}, \tau=\tau_{1}$. Then $\sigma_{C}$ is expressed as $\tau^{m_{1}} \rho^{n_{1}} \cdots \tau^{m_{s}} \rho^{n_{s}}\left(m_{i}, n_{i} \in \mathbf{Z}, 1 \leq i \leq s\right)$. We have the following propositon:

\section{Proposition 2}

$$
\begin{aligned}
& f_{*}(a)=a, \\
& f_{*}(b)=\left(n_{1}+\cdots+n_{s}\right) a+b, \\
& f_{*}(c)=-\left(m_{1}+\cdots+m_{s}\right) a+c .
\end{aligned}
$$

The proof of this proposition will be given in the next section. From this proposition, we have

$$
\operatorname{Im}\left(f_{*}-i d\right)=\left(m_{1}+\cdots+m_{s}\right) \mathbf{Z} a+\left(n_{1}+\cdots+n_{s}\right) \mathbf{Z} a .
$$

Here, we use the following notation:

$$
m \mathbf{Z} a=\left\{m^{\prime} a \mid m^{\prime} \text { is a multiple of } m\right\} .
$$

We use the following notation:

$$
\begin{aligned}
\operatorname{gcd}(0, i)=|i| & \text { for any integer } i \\
\operatorname{gcd}(i, j)=\operatorname{gcd}(|i|,|j|) & \text { for non-zero integers } i, j .
\end{aligned}
$$

Let $d$ denote $\operatorname{gcd}\left(m_{1}+\cdots+m_{s}, n_{1}+\cdots+n_{s}\right)$. From (1), (2), we have

$$
\operatorname{Coker}\left(f_{*}-i d\right)=(\mathbf{Z} / d \mathbf{Z}) a \oplus \mathbf{Z} b \oplus \mathbf{Z} c .
$$

Note that in the case of $d=0$, we have $\mathbf{Z} / d \mathbf{Z}=\mathbf{Z}$, and in the case of $d \neq 0$, we have $\mathbf{Z} / d \mathbf{Z}=\mathbf{Z}_{d}$, where $\mathbf{Z}_{d}$ is a cyclic group of order $d$. Therefore we have

$$
\mathbf{Z}[(\mathbf{Z} / d \mathbf{Z}) a]= \begin{cases}\mathbf{Z}[a] & (d=0), \\ \mathbf{Z}[a] /\left\langle a^{d}-1\right\rangle & (d \geq 1),\end{cases}
$$

where $\mathbf{Z}[a]$ is the ring of polynomials on $a$, and $\mathbf{Z}[a] /\left\langle a^{d}-1\right\rangle$ is the factor ring of polynomials on $a$ classified by the ideal $\left\langle a^{d}-1\right\rangle$. Thus $L(f)^{\mathrm{Ab}}$ becomes a polynomial on $a, b, c$. 
Let $\widehat{L}(f)$ denote the reduced form of $L(f)^{\mathrm{Ab}}$ obtained by substituting 1 for $b$ and for $c$. It is clear that $\widehat{L}(f)$ is a Laurant polynomial on $a$ and the number of terms in $\widehat{L}(f)$ is a lower bound for the number of fixed points of $f$.

Definition 8 For $x \in \operatorname{Fix}(f)$, let $I(x)$ be the coefficient of $a$ in the abelianized coordinate $R(x)^{\mathrm{Ab}} \in(\mathbf{Z} / d \mathbf{Z}) a \oplus \mathbf{Z} b \oplus \mathbf{Z} c$. We call $I(x)$ the intersection number of $x$.

This number coincides, modulo $d$, with the usual notion of an intersection number of the loop $w(f \circ \ell) \ell^{-1}$ with the segment connecting $x_{1}$ to $x_{2}$. For each $[i] \in \mathbf{Z} / d \mathbf{Z}$, where $i \in \mathbf{Z}$, let $\operatorname{Fix}_{[i]}(f)$ be the set of fixed points having intersection number $[i]$, i.e., $\operatorname{Fix}_{[i]}(f)=\{x \in \operatorname{Fix}(f) \mid I(x)=[i]\}$. Then we have

$$
\widehat{L}(f)=\sum_{[i]} \operatorname{ind}\left(\operatorname{Fix}_{[i]}(f)\right) a^{[i]} \in \mathbf{Z}[(\mathbf{Z} / d \mathbf{Z}) a] .
$$

Definition 9 A Laurant polynomial $P(a) \in \mathbf{Z}[(\mathbf{Z} / d \mathbf{Z}) a]$ is called symmetric if there exists an integer $\epsilon$ which satisfies the following equality:

$$
P(a) \equiv a^{2 \epsilon} P\left(a^{-1}\right) \quad\left(\bmod a^{d} \equiv 1\right),
$$

in other words, $P(a)$ is symmetric if it is written as $P(a)=a^{\epsilon} Q(a)$, where $Q(a) \equiv Q\left(a^{-1}\right)\left(\bmod a^{d} \equiv 1\right)$. We call $[\epsilon] \in \mathbf{Z} / d \mathbf{Z}$ the center of the polynomial $P(a)$.

Assume that a braid $\sigma$ is written as a product of $\rho^{ \pm 1}$ or $\tau^{ \pm 1}$ i.e., $\sigma=$ $\tau^{m_{1}} \rho^{n_{1}} \cdots \tau^{m_{s}} \rho^{n_{s}}\left(m_{i}, n_{i} \in \mathbf{Z}, 1 \leq i \leq s\right)$. We define a non-negative integer $d(\sigma)$ as follows:

$$
d(\sigma)=\operatorname{gcd}\left(m_{1}+\cdots+m_{s}, n_{1}+\cdots+n_{s}\right) .
$$

Assume $d(\sigma) \neq 1$. If $s \geq 2$, we define an integer $\epsilon(\sigma)$ as follows:

$$
\epsilon(\sigma)=-\sum_{k=2}^{s} m_{k}\left(\sum_{l=1}^{k-1} n_{l}\right) .
$$

If $s=1$, let an integer $\epsilon(\sigma)=0$.

Theorem Let $h: T^{2} \rightarrow T^{2}$ be a homeomorphism isotopic to the identity map, and let $x_{1}, x_{2}$ be distinct fixed points of $h$. Suppose that $h$ is differentiable at $x_{i}$, and the derivatives $\mathrm{Dh}\left(x_{i}\right)$ are non-singular $(i=1,2)$. Set $C=$ 
$\left\{x_{1}, x_{2}\right\}$ and $M=T^{2}-C$. Let $X$ be the compactification of $M$ by blowing up $x_{1}$ and $x_{2}$, and $f: X \rightarrow X$ the extention of $h: M \rightarrow M$. Assume $d\left(\sigma_{C}\right) \neq 1$. Then $\widehat{L}(f)$ is a symmetric polynomial with center $\left[\epsilon\left(\sigma_{C}\right)\right]$.

\section{Example}

(A) Let $\sigma_{C}=\tau^{4} \rho \tau^{-6} \rho^{-3} \tau^{2} \rho^{2}$, then $d\left(\sigma_{C}\right)=0$ and $\epsilon\left(\sigma_{C}\right)=10$.

We have

$$
\begin{aligned}
\widehat{L}(f)= & a^{10}\left(2 a^{7}+a^{6}-2 a^{5}-18 a^{4}+29 a^{3}-2 a^{2}-49 a+76\right. \\
& \left.-49 a^{-1}-2 a^{-2}+29 a^{-3}-18 a^{-4}-2 a^{-5}+a^{-6}+2 a^{-7}\right) .
\end{aligned}
$$

(B) Let $\sigma_{C}=\tau^{-1} \rho^{3} \tau^{2} \rho^{-2} \tau \rho \tau^{2} \rho^{6}$, then $d\left(\sigma_{C}\right)=4$ and $\epsilon\left(\sigma_{C}\right)=-11$.

We have

$\widehat{L}(f)=a^{[1]}\left(-52 a^{[2]}+128 a^{[1]}-186 a^{[0]}+128 a^{[-1]}-52 a^{[-2]}\right)\left(\bmod a^{4} \equiv\right.$ $1)$.

(C) Let $\sigma_{C}=\tau^{3} \rho^{-2} \tau^{-1} \rho \tau^{4} \rho^{3} \tau^{-2} \rho^{-4} \tau \rho^{2}$, then $d\left(\sigma_{C}\right)=5$ and $\epsilon\left(\sigma_{C}\right)=8$.

We have

$\widehat{L}(f)=a^{[3]}\left(-71 a^{[2]}+199 a^{[1]}-258 a^{[0]}+199 a^{[-1]}-71 a^{[-2]}\right)\left(\bmod a^{5} \equiv\right.$ $1)$.

Remark Theorem asserts that there are some relations among fixed point indices as follows:

In the case of $d\left(\sigma_{C}\right)=0$, $\operatorname{ind}\left(\operatorname{Fix}_{\epsilon\left(\sigma_{C}\right)-i}(f)\right)=\operatorname{ind}\left(\operatorname{Fix}_{\epsilon\left(\sigma_{C}\right)+i}(f)\right)$ for any positive integer $i$.

In the case of $d\left(\sigma_{C}\right) \geq 2$, $\operatorname{ind}\left(\operatorname{Fix}_{\left[\epsilon\left(\sigma_{C}\right)-i\right]}(f)\right)=\operatorname{ind}\left(\operatorname{Fix}_{\left[\epsilon\left(\sigma_{C}\right)+i\right]}(f)\right)$ for any positive integer $i$.

\section{The Jacobian matrix and Lefschetz numbers}

We first review some facts on the relation between the Jacobian matrix and fixed points obtained by Fadell and Husseini [5], Huang and Jiang [6]. Fadell and Husseini devised a method of computing $L(f)$ for surface maps. Let $X$ be a surface with boundary, and $f: X \rightarrow X$ a continuous map. Choose a base point $x_{0}$ and a path $w$ from $x_{0}$ to $f\left(x_{0}\right)$. Choose a free basis $\left\{a_{1}, \ldots, a_{n}\right\}$ for $\pi_{1}\left(X, x_{0}\right)$. For $\varphi \in$ Aut $\pi_{1}\left(X, x_{0}\right)$, let

$$
J(\varphi)=\left(\frac{\partial \varphi\left(a_{i}\right)}{\partial a_{j}}\right)_{1 \leq i, j \leq n}
$$


be the Jacobian matrix in Fox calculus. This is an $n \times n$ matrix in $\mathbf{Z} \pi_{1}\left(X, x_{0}\right)$, the group ring of $\pi_{1}\left(X, x_{0}\right)$. Fadell and Husseini [5] proved that the element [1] $-\left[\operatorname{tr}\left(J\left(f_{\pi}\right)\right)\right]$ of $\mathbf{Z} R(f)$ coincides with $L(f)$.

Let $f: X \rightarrow X$ be the extension of $h: M \rightarrow M$. We follow the notations in the previous sections and in [6]. Recall that $\pi_{1}\left(X, x_{0}\right)$ is a free group of rank 3 generated by $a_{1}, b, c$, and $\Lambda$ is identified with $\mathbf{Z} H_{1}(X)$. Define a map $B:$ Aut $\pi_{1}\left(X, x_{0}\right) \rightarrow \mathrm{GL}(3, \Lambda)$ by

$$
B(\varphi)=J(\varphi)^{\mathrm{Ab}}
$$

where $J(\varphi)$ is defined with respect to the basis $\left\{a_{1}, b, c\right\}$ and Ab denote the abelianization operator of the group ring $\mathbf{Z} \pi_{1}\left(X, x_{0}\right)$.

We can write down the automorphisms $\rho^{ \pm 1}, \tau^{ \pm 1}$ in terms of the basis $\left\{a_{1}, b, c\right\}$ as follows [9, p. 116]:

$$
\begin{aligned}
\rho:\left\{\begin{array}{rl}
a_{1} & \mapsto c a_{1} c^{-1} \\
b & \mapsto a_{1}^{-1} b \\
c & \mapsto c
\end{array} \quad, \quad \rho^{-1}:\left\{\begin{aligned}
a_{1} & \mapsto c^{-1} a_{1} c \\
b & \mapsto c^{-1} a_{1} c b, \\
c & \mapsto c
\end{aligned}\right.\right. \\
\tau:\left\{\begin{array}{rl}
a_{1} & \mapsto b a_{1} b^{-1} \\
b & \mapsto b \\
c & \mapsto a_{1} c
\end{array} \quad \tau^{-1}:\left\{\begin{array}{rl}
a_{1} & \mapsto b^{-1} a_{1} b \\
b & \mapsto b \\
c & \mapsto b^{-1} a_{1}^{-1} b c
\end{array} .\right.\right.
\end{aligned}
$$

Using (4), (5), we can consider every braid that is written as a product of $\rho^{ \pm 1}$ or $\tau^{ \pm 1}$ as an element of Aut $\pi_{1}\left(X, x_{0}\right)$. Therefore, $\sigma_{C}$ is considered as an element of Aut $\pi_{1}\left(X, x_{0}\right)$. From Proposition [6, p. 121], we can assume that $f_{\pi}=\sigma_{C}$.

Let $\mu_{C}$ stand for the projection $H_{1}(X) \rightarrow \operatorname{Coker}\left(f_{*}-i d\right)$ as well as for its extension $\Lambda \rightarrow \mathbf{Z}$ Coker $\left(f_{*}-i d\right)$. Huang and Jiang [6] derived the equality:

$$
1-\operatorname{tr}\left(\mu_{C} B\left(\sigma_{C}\right)\right)=L(f)^{\mathrm{Ab}}
$$

which is easily obtained by the formula of Fadell and Husseini [5] quoted above.

Let $\nu(\varphi)$ denote the homomorphism on $H_{1}(X)$ and on $\Lambda$ induced by $\varphi \epsilon$ Aut $\pi_{1}\left(X, x_{0}\right)$. We should note that $B$ is not a homomorphism. However we have the product formula:

$$
B(\varphi \psi)=B(\varphi)^{\nu(\psi)} B(\psi) \text { for } \varphi, \psi \in \operatorname{Aut} \pi_{1}\left(X, x_{0}\right),
$$


where the superscript $\nu(\psi)$ means applying the substitution $\nu(\psi)$ to every entry of the matrix $B(\varphi)$.

Proof of Proposition 2. From (4), (5), we have:

$$
\begin{array}{rlrl}
\nu(\rho): \begin{cases}a & \mapsto a \\
b & \mapsto a+b, \\
c & \mapsto c\end{cases} & \nu\left(\rho^{-1}\right):\left\{\begin{array}{l}
a \mapsto a \\
b \mapsto-a+b . \\
c \mapsto c
\end{array}\right. \\
\nu(\tau):\left\{\begin{array}{ll}
a \mapsto a \\
b \\
c \mapsto b \\
c \mapsto-a+c
\end{array} \quad \nu\left(\tau^{-1}\right):\left\{\begin{array}{l}
a \mapsto a \\
b \mapsto b \\
c \mapsto a+c
\end{array}\right.\right.
\end{array}
$$

The proposition is proved by (8), (9).

\section{Proof of Theorem}

Definition 10 Let $m$ be an integer. Let $G_{m}$ be the set of $3 \times 3$ matrices $A=\left(x_{i j}(a)\right)_{1 \leq i, j \leq 3}$ whose elements are polynomials on $a$ satisfying the following equalities:

(i) $x_{11}(a)=1, x_{21}(a)=x_{31}(a)=0$,

(ii) $x_{i j}(a)=a^{2 m} x_{i j}\left(a^{-1}\right) \quad(i, j=2,3)$,

(iii) $x_{1 j}(a)=(a-1) \overline{x_{1 j}}(a) \quad(j=2,3)$,

where $\overline{x_{1 j}}(a)$ are polynomials on $a$,

(iv) $\operatorname{det} A=a^{2 m}$.

It is easy to verify that if $A \in G_{m}, B \in G_{n}$, then $A B \in G_{m+n}, A^{-1} \in$ $G_{-m}$.

For a braid $\sigma$ which is expressed as a product of $\rho^{ \pm 1}$ or $\tau^{ \pm 1}$, let $B^{\prime}(\sigma)$ denote the simplified matrix of $B(\sigma)$ obtained by substituting 1 for $b$ and for $c$, i.e., $B^{\prime}(\sigma)=\left.B(\sigma)\right|_{b=c=1}$.

Lemma 1 Let $\sigma=\rho^{r} \tau^{m} \rho^{n} \tau^{-m} \rho^{-n} \rho^{-r}$, where $m, n, r \in \mathbf{Z}$. Then $B^{\prime}(\sigma) \in$ $G_{m n}$.

Proof. From (4), (5), the matrix $B$ for the automorphisms $\rho^{ \pm 1}, \tau^{ \pm 1}$ : $\pi_{1}\left(X, x_{0}\right) \rightarrow \pi_{1}\left(X, x_{0}\right)$ become $[9$, p. 117$]$ 


$$
\begin{aligned}
B(\rho) & =\left(\begin{array}{ccc}
c & 0 & a^{-1}(a-1) \\
-a & a & 0 \\
0 & 0 & 1
\end{array}\right) \\
B\left(\rho^{-1}\right) & =\left(\begin{array}{ccc}
c^{-1} & 0 & a^{-1} c^{-1}(1-a) \\
c^{-1} & a^{-1} & a^{-1} c^{-1}(1-a) \\
0 & 0 & 1
\end{array}\right) \\
B(\tau) & =\left(\begin{array}{ccc}
b & a^{-1}(a-1) & 0 \\
0 & 1 & 0 \\
1 & 0 & a^{-1}
\end{array}\right), \\
B\left(\tau^{-1}\right) & =\left(\begin{array}{ccc}
b^{-1} & a^{-1} b^{-1}(1-a) & 0 \\
0 & 1 & 0 \\
-a b^{-1} & b^{-1}(a-1) & a
\end{array}\right) .
\end{aligned}
$$

These expressions and the product formula (7) enable one to calculate $B(\sigma)$. Here, we shall calculate in the case of $m, n, r \in \mathbf{N}$. Let $\left.B\left(\rho^{r}\right)^{\nu\left(\tau^{m} \rho^{n} \tau^{-m} \rho^{-n} \rho^{-r}\right)} B\left(\tau^{m} \rho^{n}\right)^{\nu\left(\tau^{-m} \rho^{-n} \rho^{-r}\right)}\right|_{b=c=1}$ denote $\left(\alpha_{i j}(a)\right)$, and let $\left.B\left(\tau^{-m} \rho^{-n}\right)^{\nu\left(\rho^{-r}\right)} B\left(\rho^{-r}\right)\right|_{b=c=1}$ denote $\left(\beta_{i j}(a)\right)$. To avoid complexity, we use abbreviation as follows:

$$
A_{n}^{m}=\sum_{k=1}^{n} a^{k m}
$$

Then, we have

$$
\begin{aligned}
& \alpha_{11}=a^{m(n-r)}-a^{-m r}(a-1)\left\{a^{n-m}(r+1) A_{m}^{n} A_{n}^{m-1}\right. \\
& \left.-r\left(a^{n-m+1} A_{n}^{m-1}+a^{m n}\right) A_{m}^{r-1}\right\} \\
& \alpha_{12}=a^{n+r-1}(a-1)\left\{A_{m}^{-r}+r a^{-1}(a-1) \sum_{k=1}^{m-1} A_{k}^{1-r} a^{-k}\right\} \\
& \alpha_{13}=a^{-(m+1)(r+1)}(a-1)\left\{r a^{r(m+1)}-a^{n+r} A_{m}^{r} A_{n}^{m-1}+r a^{r}(a-1)\right. \\
& \left.\times\left(a A_{m}^{r-1} \sum_{k=1}^{n-1} A_{k}^{m-1} a^{k}+A_{m}^{r-1} A_{n}^{m}-A_{m}^{r} \sum_{k=1}^{n-1} A_{k}^{m-1} a^{k}\right)+A_{m+1}^{r} A_{n}^{m}\right\}
\end{aligned}
$$




$$
\begin{aligned}
& \alpha_{21}=a^{-m(r+1)}\left\{a^{m(n+1)}\left(a r A_{m}^{r-1}-A_{r}^{1}\left(A_{m}^{r-1}+1\right)\right)-a^{(m+1) r+n+1}\right. \\
& \left.\times A_{n}^{m-1}+a^{n+1} A_{n}^{m-1}\left(a r A_{m}^{r-1}-A_{m}^{r-1} A_{r}^{1}+A_{m}^{r}\left(A_{r}^{1}-r\right)\right)\right\}, \\
& \alpha_{22}=a^{n+r}-a^{n}(a-1)\left\{a^{-(m r+1)} A_{m}^{r} A_{r}^{1}+a^{r-1}\left(a^{-1} A_{r}^{1}-r\right)\right. \\
& \left.\times \sum_{k=1}^{m-1} A_{k}^{1-r} a^{-k}\right\} \\
& \alpha_{23}=-a^{-(m r+m+1)}(a-1) A_{r}^{1}\left(a^{-r} A_{m+1}^{r} A_{n}^{m}-a^{n} A_{m}^{r} A_{n}^{m-1}\right) \\
& +a^{r-m}\left(A_{n-1}^{m}-a^{n} A_{n-1}^{m-1}\right)+a^{-m}\left(r-a^{-1} A_{r}^{1}\right) \\
& \times\left\{(a-1)\left\{a^{-m r}\left(A_{m}^{r-1} A_{n}^{m}+\left(a A_{m}^{r-1}-A_{m}^{r}\right) \sum_{k=1}^{n-1} A_{k}^{m-1} a^{k}\right)\right\}+1\right\}, \\
& \alpha_{31}=a^{1-m r}\left\{a^{n-m} A_{n}^{m-1}\left(a A_{m}^{r-1}-A_{m}^{r}\right)+a^{m n} A_{m}^{r-1}\right\} \\
& \alpha_{32}=a^{n+r-1}(a-1) \sum_{k=1}^{m-1} A_{k}^{1-r} a^{-k} \\
& \alpha_{33}=a^{-m}\left\{(a-1)\left\{a^{-m r}\left(A_{m}^{r-1} A_{n}^{m}+\left(a A_{m}^{r-1}-A_{m}^{r}\right) \sum_{k=1}^{n-1} A_{k}^{m-1} a^{k}\right)\right\}+1\right\} \\
& \beta_{11}=1 \text {, } \\
& \beta_{12}=-a^{n+r+1}(a-1) A_{m}^{n+r} \text {, } \\
& \beta_{13}=a^{-1}(a-1)\left\{\left(n+r-a^{-(n+1)}\left(a^{-r} A_{r}^{1}+A_{n}^{1}\right)\right) A_{m}^{n+r}\right. \\
& \left.-(n+r) a^{m(n+r)}\right\} \\
& \beta_{21}=a^{-(n+r)} A_{n+r}^{1}, \\
& \beta_{22}=a^{-(n+r)} \text {, } \\
& \beta_{23}=a^{-(n+r+1)} A_{n+r}^{1}-(n+r), \\
& \beta_{31}=-A_{m}^{1} \\
& \beta_{32}=a^{-(n+r)}(a-1) \sum_{k=1}^{m} A_{k}^{n+r-1} a^{k}, \\
& \beta_{33}=(a-1)\left\{r a^{-1}\left(a^{-n} A_{m}^{n+r} A_{n}^{1}-a^{m+1}\left(a^{-n} A_{n}^{1}-1\right) A_{m}^{n+r-1}\right)\right.
\end{aligned}
$$




$$
\begin{array}{r}
+a^{-n}\left(a^{-(r+1)} A_{r}^{1}-r\right) \sum_{k=1}^{m} A_{k}^{n+r-1} a^{k}+a^{m} A_{m}^{n+r-1}\left(n-a \sum_{k=1}^{n} A_{k}^{-1}\right) \\
\left.+A_{m}^{n+r} \sum_{k=1}^{n} A_{k}^{-1}\right\}+a^{m}
\end{array}
$$

Denote $B^{\prime}(\sigma)=\left(x_{i j}(a)\right)_{1 \leq i, j \leq 3}$. Since $x_{i j}(a)=\sum_{k=1}^{3} \alpha_{i k} \beta_{k j}(i, j=1,2,3)$, we can show that $B^{\prime}(\sigma) \bar{\in} G_{m n}$ by calculating straightforwardly. We can also prove the lemma in the case of $m, n, r \in \mathbf{Z}$ by a similar argument described above.

Now, we need the following lemma which is a generalization of results in $[9$, p. 120].

Lemma 2 Let $\sigma=\tau^{m_{1}} \rho^{n_{1}} \cdots \tau^{m_{s}} \rho^{n_{s}}$, where $m_{1}+\cdots+m_{s}=n_{1}+\cdots+$ $n_{s}=0\left(m_{i}, n_{i} \in \mathbf{Z}, 1 \leq i \leq s, s \geq 2\right)$. Then $B^{\prime}(\sigma) \in G_{\epsilon(\sigma)}$.

Proof. We shall prove the lemma by induction.

Case 1: Consider the case of $s=2$. In this case, the lemma is a special case of Lemma 1 of $r=0$ since $\epsilon(\sigma)=-m_{2} n_{1}=m_{1} n_{1}$.

Case 2: Consider the case of $s=3$, i.e., $\sigma=\tau^{m_{1}} \rho^{n_{1}} \tau^{m_{2}} \rho^{n_{2}} \tau^{m_{3}} \rho^{n_{3}}$, where $m_{1}+m_{2}+m_{3}=n_{1}+n_{2}+n_{3}=0$. Let $\sigma_{1}, \sigma_{2}$ denote $\tau^{m_{1}} \rho^{n_{1}} \tau^{-m_{1}} \rho^{-n_{1}}$, $\rho^{n_{1}}\left(\tau^{m_{1}+m_{2}} \rho^{n_{2}} \tau^{m_{3}} \rho^{n_{1}+n_{3}}\right) \rho^{-n_{1}}$ respectively. From Lemma 1 we have $B^{\prime}\left(\sigma_{1}\right) \in G_{m_{1} n_{1}}$ and $B^{\prime}\left(\sigma_{2}\right) \in G_{\left(m_{1}+m_{2}\right) n_{2}}$. Since $\sigma=\sigma_{1} \sigma_{2}$, we obtain

$$
B^{\prime}(\sigma)=B^{\prime}\left(\sigma_{1}\right) B^{\prime}\left(\sigma_{2}\right) \in G_{m_{1} n_{1}+\left(m_{1}+m_{2}\right) n_{2}}=G_{\epsilon(\sigma)} .
$$

Case 3: Now, suppose that the lemma is proved for all $s(2 \leq s \leq p, p \geq$ $3)$. We shall prove the lemma in the case of $s=p+1$, i.e., $\sigma=\tau^{m_{1}} \rho^{n_{1}} \ldots$ $\tau^{m_{p+1}} \rho^{n_{p+1}}$, where $m_{1}+\cdots+m_{p+1}=n_{1}+\cdots+n_{p+1}=0$. To avoid complexity, we use abbreviation as follows:

$$
M=\sum_{k=1}^{p-1} m_{k}, \quad N=\sum_{k=1}^{p-1} n_{k}
$$

Let $\sigma_{1}, \sigma_{2}$ and $\sigma_{3}$ denote $\tau^{m_{1}} \rho^{n_{1}} \cdots \tau^{m_{p-1}} \rho^{n_{p-1}} \tau^{-M} \rho^{-N}, \tau^{M} \rho^{N} \tau^{-M} \rho^{-N}$ and $\tau^{M} \rho^{N} \tau^{m_{p}} \rho^{n_{p}} \tau^{m_{p+1}} \rho^{n_{p+1}}$ respectively. From the hypotheses of induction, we have $B^{\prime}\left(\sigma_{1}\right) \in G_{\epsilon\left(\sigma_{1}\right)}, B^{\prime}\left(\sigma_{2}\right)^{-1} \in G_{-M N}$, and $B^{\prime}\left(\sigma_{3}\right) \in G_{\epsilon\left(\sigma_{3}\right)}$. 
Hence,

$$
B^{\prime}\left(\sigma_{1}\right) B^{\prime}\left(\sigma_{2}\right)^{-1} B^{\prime}\left(\sigma_{3}\right) \in G_{\epsilon\left(\sigma_{1}\right)-M N+\epsilon\left(\sigma_{3}\right)} .
$$

Therefore, since $\epsilon\left(\sigma_{1}\right)-M N+\epsilon\left(\sigma_{3}\right)=\epsilon(\sigma)$ and

$$
B^{\prime}(\sigma)=B^{\prime}\left(\sigma_{1}\right) B^{\prime}\left(\sigma_{2}\right)^{-1} B^{\prime}\left(\sigma_{3}\right),
$$

we have $B^{\prime}(\sigma) \in G_{\epsilon(\sigma)}$. This completes the proof of the lemma.

Proof of Theorem, Recall that $\sigma_{C}=\tau^{m_{1}} \rho^{n_{1}} \cdots \tau^{m_{s}} \rho^{n_{s}} \quad\left(m_{i}, n_{i} \in \mathbf{Z}, 1 \leq\right.$ $i \leq s)$. For brevity, we shall write $d=d\left(\sigma_{C}\right)$. Let $\mu_{C}^{\prime}: \mathbf{Z}[a] \rightarrow \mathbf{Z}[a] /\left\langle a^{d}-1\right\rangle$ be the natural projection. From (3), (6), we have

$$
1-\operatorname{tr}\left(\mu_{C}^{\prime} B^{\prime}\left(\sigma_{C}\right)\right)=\widehat{L}(f) .
$$

Therefore, to prove the theorem, we have only to show that $1-\operatorname{tr}\left(\mu_{C}^{\prime} B^{\prime}\left(\sigma_{C}\right)\right)$ is symmetric.

In the case of $d=0$, then $\mu_{C}^{\prime} B^{\prime}\left(\sigma_{C}\right)=B^{\prime}\left(\sigma_{C}\right)$ and the theorem is easily proved from Lemma 2.

We shall prove the theorem in the case of $d \geq 2$.

Case 1: First, we consider the case of $s=1$. For $n \in \mathbf{Z}$, we define an integer $s(n)$ as 1,0 , and -1 in the case of $n>0, n=0$, and $n<0$ respectively. For brevity, we shall write $m=m_{1}, n=n_{1}$. Then we have

$$
\mu_{C}^{\prime} B^{\prime}\left(\sigma_{C}\right)=\left(\begin{array}{ccc}
1 & m a^{-1}(a-1) & n(m+1) a^{-1}(a-1) \\
-\mathrm{s}(n) A_{|n|}^{1} & 1 & n-\mathrm{s}(n) A_{|n|}^{1} \\
\mathrm{~s}(m) A_{|m|}^{1} & m-\mathrm{s}(m) A_{|m|}^{1} & m n+1-\mathrm{s}(m n)|n| A_{|m|}^{1}
\end{array}\right) .
$$

Then $\widehat{L}(f)$ is a symmetric polynomial with center $[0] \in \mathbf{Z}_{d}$ since $a^{i}(a+\cdots+$ $\left.a^{|m|}\right) \equiv a+\cdots+a^{|m|}\left(\bmod a^{d} \equiv 1\right)$ for any $i \in \mathbf{Z}$.

Case 2: Secondly, we consider the case of $s \geq 2$. Let

$$
\begin{gathered}
\sigma_{1}=\tau^{m_{1}} \rho^{n_{1}} \cdots \tau^{m_{s}} \rho^{n_{s}} \tau^{-M} \rho^{-N}, \quad \sigma_{2}=\tau^{M} \rho^{N} \tau^{-M} \rho^{-N} \\
\text { and } \sigma_{3}=\tau^{M} \rho^{N},
\end{gathered}
$$

where $M=\sum_{k=1}^{s} m_{k}, N=\sum_{k=1}^{s} n_{k}$, then we have

$$
B^{\prime}\left(\sigma_{C}\right) \equiv B^{\prime}\left(\sigma_{1}\right) B^{\prime}\left(\sigma_{2}\right)^{-1} B^{\prime}\left(\sigma_{3}\right) \quad\left(\bmod a^{d} \equiv 1\right) .
$$


By Lemma 2, we have

$$
B^{\prime}\left(\sigma_{1}\right) \in G_{\epsilon\left(\sigma_{1}\right)}, \quad B^{\prime}\left(\sigma_{2}\right)^{-1} \in G_{-M N} .
$$

Since $\mu_{C}^{\prime} B^{\prime}\left(\sigma_{3}\right)$ can be obtained as in Case 1 by substituting $\sigma_{3}$ for $\sigma_{C}$, we have from (11) that

$$
B^{\prime}\left(\sigma_{1}\right) B^{\prime}\left(\sigma_{2}\right)^{-1} B^{\prime}\left(\sigma_{3}\right) \equiv\left(\begin{array}{ccc}
1 & * & * \\
* & x_{22}(a) & x_{23}(a) \\
* & x_{32}(a) & x_{33}(a)
\end{array}\right) \quad\left(\bmod a^{d} \equiv 1\right)
$$

where $x_{i j}(a)=a^{2 \epsilon\left(\sigma_{C}\right)} x_{i j}\left(a^{-1}\right)(i, j=2,3)$.

From (10), (12), we can obtain the consequence of Theorem in the case of $d \geq 2$, because $x_{i j}(a)$ are symmetric polynomials with center $\left[\epsilon\left(\sigma_{C}\right)\right]$ $(i, j=2,3)$. We complete the proof of Theorem.

Acknowledgements The author wishes to thank Professor Takashi Matsuoka, of Naruto University of Education, who contributed many significant suggestions and advices and much invaluable encouragement. $\mathrm{He}$ also would like to express his thanks to the referee whose suggestions led to an improvement of the paper.

\section{References}

[1] Birman J.S., On braid groups. Comm. Pure Appl. Math. 22 (1969), 41-72.

[2] Birman J.S., Braids, Links, and Mapping Class Groups. Ann. Math. Studies Vol. 82, Princeton Univ. Press, Princeton, 1974.

[3] Bowen R., Entropy and the fundamental group. In "Structure of Attractors in Dynamical Systems", Markley N.G., et al. (eds.), Lecture Notes in Math. 668, Springer, Berlin, Heidelberg, New York (1978), 21-29.

[4] Brown R.F., The Lefschetz Fixed Point Theorem. Scott, Foresman and Company, Glenview, 1971.

[5] Fadell E. and Husseini S., The Nielsen number on surfaces. Topological Methods in Nonlinear Functional Analysis (Singh S.P., et al., eds.), Contemp. Math. 21, Amer. Math. Soc., Providence (1983), 59-98.

[6] Huang H.H. and Jiang B.J., Braids and periodic solutions. Topological Fixed Point Theory and Applications (Jiang B.J., ed.), Lecture Notes in Math. 1411, SpringerVerlag, Berlin, Heidelberg, New York (1989), 107-123.

[ 7 ] Jiang B.J., Lectures on Nielsen Fixed Point Theory. Contemp. Math. Vol. 14, Amer. Math. Soc., Providence, 1983. 
[8] Moran S., The Mathematical Theory of Knots and Braids; an Introduction. NorthHolland Math. Studies Vol. 82, North-Holland, Amsterdam, 1983.

[9] Shiraki H., On braid type of fixed points of homeomorphisms defined on the torus. Memoirs Fac. Sci. Kochi Univ. 20 (1999), 113-122.

Department of General Education Kochi National College of Technology

Nankoku-shi, Kochi 783-8508

Japan

E-mail: shiraki@ge.kochi-ct.ac.jp 